\title{
A practical formula for the radiated angular momentum
}

\author{
Carlos O. Lousto and Yosef Zlochower \\ Center for Computational Relativity and Gravitation, \\ School of Mathematical Sciences, Rochester Institute of Technology, \\ 78 Lomb Memorial Drive, Rochester, New York 14623
}

(Dated: June 18, 2021)

\begin{abstract}
We present a simple formula for the radiated angular momentum based on a spin-weighted spherical harmonic decomposition of the Weyl scalar $\psi_{4}$ representing outgoing radiation in the Kinnersley tetrad. We test our formula by measuring the radiated angular momentum from three simulations of non-spinning equal-mass black-hole binaries with orbital angular momentum aligned along the $x$, $y$, and $z$ axes respectively. We find that the radiated angular momentum agrees with the differences in the remnant horizon spins and the initial angular momentum for each system.

PACS numbers: 04.25.Dm, 04.30.Db, 04.70.Bw, 04.30.-w
\end{abstract}

\section{INTRODUCTION}

Recent breakthroughs in numerical relativity [1, 2, 3] have made it possible to accurately evolve black-hole black-hole [4, [5, 6, 7] and black-hole - neutron-star [8, 9] binaries for many orbits. The feasibility of evolving generic black-hole binaries was demonstrated in [10] where unexpectedly large recoil velocities of the merger remnant [10, 11] were found. One of the important methods to measure the accuracy of the full numerical evolutions is to monitor the conservation of the energy, linear momentum, and angular momentum. A practical way of doing this in black-hole binary systems is to compute the final black hole mass, linear momentum, and angular momentum from the (isolated) horizon expressions, as well the radiated energy, linear momentum, and angular momentum as measured far away from the system (formally at null infinity, $\mathscr{I}^{+}$). The sum of the corresponding remnant and radiated quantities should converge to the initial mass, linear momentum, and angular momentum of the system.

Expressions for the energy and momenta of an isolated system have long been established (see Refs. 12, 13] for reviews). Currently, the most popular method to extract gravitational radiation is to compute the Weyl scalar $\psi_{4}$ in a quasi-Kinnersley frame [14] or a frame that reduces to one asymptotically. For practical purposes, it is then convenient to express the radiation formulae for the mass, linear momentum, and angular momentum in terms of $\psi_{4}$. That was done in Ref. [15], see formulae (22)-(24) there, where the radiated angular momentum was only given along the $\mathrm{z}$-axis. We extend the computation of the radiated angular momentum to all the three Cartesian components. These are useful to verify the conservation of the angular momentum in generic black-hole binaries, for instance, when spin precession is present [10, 11, 16].

\section{DERIVATION}

The angular momentum on a space-like slice $\Sigma^{+}$of $\mathscr{I}^{+}$ (e.g. the intersection of a $u=t-r=$ const slice with $\mathscr{I}^{+}$) is given by [13]

$J_{[i]}=\frac{1}{16 \pi} \operatorname{Re}\left\{\oint_{\Sigma^{+}} d S \xi_{[i]}^{A} \bar{q}_{A}\left[2 \psi_{1}^{0}-2 \sigma^{0} \precsim \bar{\sigma}^{0}-ð\left(\sigma^{0} \bar{\sigma}^{0}\right)\right]\right\}$,

where the ${ }^{0}$ superscript indicates the coefficient of the leading-order term in an expansion in $1 / r$, the spincoefficient $\sigma$ and Weyl scalars $\psi_{0}, \cdots, \psi_{4}$ are given in terms of a null tetrad $\left(l^{a}, n^{a}, m^{a}, \bar{m}^{a}\right)$ by (Eqs. (3.7-3.8) of Ref. [17])

$$
\begin{aligned}
\sigma & =m^{b} m^{a} \nabla_{a} n_{b}, \\
\psi_{0} & =-C_{a b c d} l^{a} m^{b} l^{c} m^{d}, \\
\psi_{1} & =-C_{a b c d} l^{a} n^{b} l^{c} m^{d}, \\
\psi_{2} & =-\frac{1}{2}\left(C_{a b c d} l^{a} n^{b} l^{c} n^{d}-C_{a b c d} l^{a} n^{b} m^{c} \bar{m}^{d}\right), \\
\psi_{3} & =C_{a b c d} l^{a} n^{b} n^{c} \bar{m}^{d}, \\
\psi_{4} & =-C_{a b c d} n^{a} \bar{m}^{b} n^{c} \bar{m}^{d},
\end{aligned}
$$

(where $C_{a b c d}$ is the Weyl tensor), and have the asymptotic form (Eq. (4.4) in [17]):

$$
\begin{aligned}
\sigma & =\sigma^{0} r^{-2}+\mathcal{O}\left(r^{-3}\right), \\
\psi_{0} & =\psi_{0}^{0} r^{-5}+\mathcal{O}\left(r^{-6}\right), \\
\psi_{1} & =\psi_{1}^{0} r^{-4}+\mathcal{O}\left(r^{-5}\right), \\
\psi_{2} & =\psi_{2}^{0} r^{-3}+\mathcal{O}\left(r^{-4}\right), \\
\psi_{3} & =\psi_{3}^{0} r^{-2}+\mathcal{O}\left(r^{-3}\right), \\
\psi_{4} & =\psi_{4}^{0} r^{-1}+\mathcal{O}\left(r^{-2}\right),
\end{aligned}
$$

the coordinates on the slice $\Sigma^{+}$are such that the volume element has the standard form for a 2 -sphere, $d S=$ $\sin \theta d \theta d \phi$, the capital $A$ index ranges from 1 to 2 over the angular coordinates in the slice, the dyad $q^{A}$ is the standard unit-sphere dyad $\left(q^{A}=(1, i / \sin \theta)\right.$ in $(\theta, \phi)$ coordinates), the index $i$ ranges from 1 to 3 and represents 
the three Cartesian components of the angular momentum, an overbar indicates complex conjugation, the three $\xi_{[i]}^{A}$ are rotational Killing vectors intrinsic to $\Sigma^{+}$, and the $\widetilde{\partial}$ and $\bar{\gamma}$ operators acting a function $v_{s}$ of spin-weight $s$ have the form

$$
\begin{aligned}
& \partial v_{s}=-\partial_{\theta} v_{s}-\frac{i}{\sin \theta} \partial_{\phi} v_{s}+s v_{s} \cot \theta \\
& \bar{\partial} v_{s}=-\partial_{\theta} v_{s}+\frac{i}{\sin \theta} \partial_{\phi} v_{s}-s v_{s} \cot \theta
\end{aligned}
$$

The tetrad used in Eq. (1) is partially fixed by the choices $l_{a}=\nabla_{a} u, u=(t-r) / \sqrt{2}+\mathcal{O}(\log r)$, and $m^{a}=(0,0,1, i / \sin \theta) / \sqrt{2}+\mathcal{O}(1 / r)$, where $r$ is a luminosity distance (we consider here the case where the spacetime and coordinates are such that the metric approaches $\operatorname{diag}(1,1,1)$ at large $r$ and hence the coordinate $r$ is a luminosity distance). Note that $l^{a}$ must therefore be hypersurface orthogonal. The quasi-Kinnersley $l_{a}$, by contrast, is not hypersurface orthogonal when the spin of the hole is non-vanishing and will give an incorrect value for the angular momentum (e.g. zero for a Kerr black hole). However, as we will derive the radiated angular momentum in terms of $\psi_{4}$, and the Kinnersley [18] $l_{a}$ differs from $\nabla_{a} u$ by a type II rotation (which leaves $\psi_{4}$ invariant) and a trivial boost of $\sqrt{2}$, the Kinnersley $\psi_{4}$ can be used for the radiated angular momentum formulae derived below since it is invariant under type II rotations.

Note that for any spin-zero function $f$ and Killing vector (of the unit-sphere metric) $\xi^{A}$,

$\left.\operatorname{Re}\left\{\oint_{\Sigma^{+}} d S \xi^{A} \bar{q}_{A} \partial f\right\}=\operatorname{Re}\left\{\oint_{\Sigma^{+}} d S \xi^{A} \bar{q}_{A} \frac{1}{2} \check{\partial}(f-\bar{f})\right]\right\}$,

i.e. only the imaginary part of $f$ contributes to the integral. Thus the final term in Eq. (1) does not contribute to $J$.

Let us define $\Phi_{[i]}=\xi_{[i]}^{A} q_{A}$ where $\xi_{[i]}^{A}$ are the standard rotational Killing vectors about the $x, y$, and $z$ axes respectively, then

$$
\begin{aligned}
& \Phi_{[x]}=i \cos \theta \cos \phi-\sin \phi=2 i \sqrt{\frac{\pi}{3}}\left({ }_{-1} Y_{11}-{ }_{-1} Y_{1-1}\right), \\
& \Phi_{[y]}=i \cos \theta \sin \phi+\cos \phi=2 \sqrt{\frac{\pi}{3}}\left({ }_{-1} Y_{11}+{ }_{-1} Y_{1-1}\right) \\
& \Phi_{[z]}=-i \sin \theta=-2 i \sqrt{\frac{2 \pi}{3}}-1 Y_{10},
\end{aligned}
$$

where the spin-weighted spherical harmonics have the form [21]

$$
\begin{aligned}
& { }_{s} Y_{\ell m}=(-1)^{s} \sqrt{\frac{(\ell-s) !}{(\ell+s) !}} \partial^{s} Y_{\ell m} \quad(\text { for } s \geq 0), \\
& { }_{s} Y_{\ell m}=\sqrt{\frac{(\ell+s) !}{(\ell-s) !} \overline{\mathrm{o}}^{-s} Y_{\ell m} \quad(\text { for } s \leq 0) .}
\end{aligned}
$$

Note that all three $\Phi_{[i]}$ can be expressed as a sum of $\ell=1$ spin-weighted spherical harmonics (of spin-weight -1 ). Hence the angular momentum loss is given by

$$
\dot{J}_{[i]}=\frac{1}{16 \pi} \operatorname{Re}\left\{\oint_{\Sigma^{+}} d S \Phi_{[i]}\left(2 \dot{\psi}_{1}^{0}-2 \dot{\sigma}^{0} \partial \bar{\sigma}^{0}-2 \sigma^{0} \partial \dot{\bar{\sigma}}^{0}\right)\right\},
$$

where a dot indicates differentiation with respect to $u$. Using the following relations [17],

$$
\begin{aligned}
\dot{\psi}_{1}^{0} & =-\check{\mathrm{\partial}} \psi_{2}^{0}+2 \sigma^{0} \dot{\mathrm{\sigma}}^{0}, \\
\psi_{2}^{0}-\bar{\psi}_{2}^{0} & =\overline{\mathrm{\partial}}^{2} \sigma^{0}-\check{\partial}^{2} \bar{\sigma}^{0}+\bar{\sigma}^{0} \dot{\sigma}^{0}-\sigma^{0} \dot{\bar{\sigma}}^{0}, \\
\psi_{4}^{0} & =-\ddot{\bar{\sigma}}^{0},
\end{aligned}
$$

Eq. (18) reduces to (where we used result (15))

$$
\begin{aligned}
\dot{J}_{[i]}= & \frac{1}{16 \pi} \operatorname{Re}\left\{\oint _ { \Sigma ^ { + } } d S \Phi _ { [ i ] } \left(\check{\partial}^{3} \bar{\sigma}^{0}-ð \bar{\partial}^{2} \sigma^{0}-\right.\right. \\
& \left.\left.3 \dot{\sigma}^{0} \precsim \bar{\sigma}^{0}+3 \sigma^{0} \precsim \dot{\bar{\sigma}}^{0}-\bar{\sigma}^{0} \precsim \dot{\sigma}^{0}+\dot{\bar{\sigma}}^{0} \partial \sigma^{0}\right)\right\} .
\end{aligned}
$$

Note that the first two terms do not contribute to $\dot{J}_{[i]}$ since $\Phi_{[i]}$ contains only $\ell=1$ modes and $\sigma^{0}$ (which has spin-weight 2) cannot contain $\ell=1$ modes. Hence we have

$$
\begin{aligned}
\dot{J}_{[i]}= & \frac{1}{16 \pi} \operatorname{Re}\left\{\oint _ { \Sigma ^ { + } } d S \Phi _ { [ i ] } \left(-3 \dot{\sigma}^{0} \precsim \bar{\sigma}^{0}+3 \sigma^{0} \precsim \dot{\bar{\sigma}}^{0}-\right.\right. \\
& \left.\left.\bar{\sigma}^{0} \partial \dot{\sigma}^{0}+\dot{\bar{\sigma}}^{0} \precsim \sigma^{0}\right)\right\} .
\end{aligned}
$$

In terms of the more standard choice of tetrad with $l_{a}=$ $\nabla_{a}(t-r)$, the radiated angular momentum takes the form

$$
\begin{aligned}
\frac{d J_{[i]}}{d t}= & \frac{1}{16 \pi} \operatorname{Re}\left\{\oint_{\Sigma^{+}} d S \Phi_{[i]}(-3 N ð \bar{H}+3 H ð \bar{N}-\right. \\
& \bar{H} \partial N+\bar{N} \partial H)\}
\end{aligned}
$$

where $\partial_{t t} H=\partial_{t} N=\bar{\psi}_{4}$.

After expressing $H$ and $N$ in terms of spin-2 spherical harmonics

$$
\begin{aligned}
H & =\sum_{\ell, m} H_{\ell m 2} Y_{\ell m}, \\
N & =\sum_{\ell, m} N_{\ell m 2} Y_{\ell m},
\end{aligned}
$$

Eq. (24) becomes

$$
\frac{d J_{[i]}}{d t}=-\frac{1}{16 \pi} \sum_{\ell, m, \ell^{\prime}, m^{\prime}} \operatorname{Re}\left\{\bar{H}_{\ell m} N_{\ell^{\prime} m^{\prime}} d_{\ell m \ell^{\prime} m^{\prime}}^{[i]}\right\}
$$

where

$$
d_{\ell m \ell^{\prime} m^{\prime}}^{[i]}=c_{\ell m \ell^{\prime} m^{\prime}}^{[i]}-\bar{c}_{\ell^{\prime} m^{\prime} \ell m}^{[i]},
$$

and

$$
\begin{aligned}
c_{\ell m \ell^{\prime} m^{\prime}}^{[i]}= & \oint_{\Sigma^{+}} d S \Phi_{[i]}\left(3 \sqrt{(\ell+2)(\ell-1)}{ }_{1} \bar{Y}_{\ell m 2} Y_{\ell^{\prime} m^{\prime}}\right. \\
& \left.-\sqrt{\left(\ell^{\prime}-2\right)\left(\ell^{\prime}+3\right)}{ }_{2} \bar{Y}_{\ell m 3} Y_{\ell^{\prime} m^{\prime}}\right) .
\end{aligned}
$$


The coefficients $d_{\ell m \ell^{\prime} m^{\prime}}^{[i]}$ have the simple form

$$
\begin{aligned}
d_{\ell m \ell^{\prime} m^{\prime}}^{[i]}= & \delta_{\ell \ell^{\prime}}\left[2 i \sqrt{\ell(\ell+1)-m m^{\prime}} \delta_{\left|m-m^{\prime}\right| 1}\right. \\
& 2\left(m-m^{\prime}\right) \sqrt{\ell(\ell+1)-m m^{\prime}} \delta_{\left|m-m^{\prime}\right| 1} \\
& \left.4 i m \delta_{m m^{\prime}}\right]
\end{aligned}
$$

22]. The radiated angular momenta then has the form

$$
\begin{aligned}
\frac{d J_{x}}{d t}= & \frac{1}{8 \pi} \sum_{\ell m}\left(\sqrt{\ell(\ell+1)-m(m+1)} \operatorname{Im}\left\{\bar{H}_{\ell m} N_{\ell m+1}\right\}\right. \\
& \left.+\sqrt{\ell(\ell+1)-m(m-1)} \operatorname{Im}\left\{\bar{H}_{\ell m} N_{\ell m-1}\right\}\right),(31) \\
\frac{d J_{y}}{d t}= & \frac{1}{8 \pi} \sum_{\ell m}\left(\sqrt{\ell(\ell+1)-m(m+1)} \operatorname{Re}\left\{\bar{H}_{\ell m} N_{\ell m+1}\right\}\right. \\
& \left.-\sqrt{\ell(\ell+1)-m(m-1)} \operatorname{Re}\left\{\bar{H}_{\ell m} N_{\ell m-1}\right\}\right),(32) \\
\frac{d J_{z}}{d t}= & \frac{1}{4 \pi} \sum_{\ell m} m \operatorname{Im}\left\{\bar{H}_{\ell m} N_{\ell m}\right\} .
\end{aligned}
$$

Note that if $\psi_{4}^{0}=\sum P_{\ell m-2} Y_{\ell m}$, then $N_{\ell m}=$ $(-1)^{m} \int_{-\infty}^{t} \bar{P}_{\ell-m}(\tau) d \tau$, and $H_{\ell m}=\int_{-\infty}^{t} N_{\ell m}(\tau) d \tau$. In practice one needs to integrate from the starting time of the simulation rather than $t=-\infty$. This presents no significant problem for black-hole binary simulations as the error introduced by assuming no radiation in the past is of the same order as the error introduced by using initial data without the correct radiation content. In addition, these simulation typically start when the radiation is quite small compared to the late-inspiral and merger waveforms.

\section{NUMERICAL TESTS}

We tested the newly introduced formulae for the $x$, $y$, and $z$ components of the radiated angular momentum by evolving the non-spinning, quasi-circular S0 configuration of Ref. 19] rotated such that orbital angular momentum is aligned along the $x$ (SOX), $y$ (S0Y), and $z$ (SOZ) axes respectively. For reference we provide the initial data parameters in Table I for these configurations. The total radiated angular momentum for each configuration is reported in Table [1. We ran the SOX and SOY configurations with our new AMR code [10] with 9 levels of refinement and central resolution of $M / 40$. We extracted the waveform at radii $r=25 M, 30 M, 35 M$, and $40 M$. We fit the calculated radiated angular momentum to a linear and quadratic polynomial in $1 / r$. We take the quadratic extrapolation to be the value at $r=\infty$ and the quoted errors are the differences between the linear and quadratic extrapolations. Note that we did not re-run the $\mathrm{S} 0 \mathrm{Z}$ configuration, but rather re-analyzed the waveform from an existing unigrid run with a relatively low central resolution of $h=M / 22.5$ and extraction radii at $r=20 M, 25 M$, and
TABLE I: Initial data and total radiated angular momentum for quasi-circular, equal-mass black-hole binaries. The total angular momentum is $J / M^{2}=0.8764$ and the proper horizon separation is $l / M=10.01$. The punctures are located at $\pm(X, Y, Z)$, with mass parameter $m_{p} / M=0.4848$, and momentum $\pm\left(P_{x}, P_{y}, P_{z}\right)$. The orbital parameter $(X, Y, Z)$ and $\left(P_{x}, P_{y}, P_{z}\right)$ are obtained by the $3 \mathrm{PN}$ equations of motion for equal-mass binaries with orbital frequency $M \Omega=0.0500$. The puncture masses are obtained by setting the ADM mass of the system to $(1.0000 \pm 0.0005) M$.

\begin{tabular}{llll}
\hline \hline Config & S0X & S0Y & S0Z \\
$X / M$ & 0.000 & 0.000 & 3.280 \\
$Y / M$ & 0.000 & 0.000 & 0.000 \\
$Z / M$ & 3.280 & 3.280 & 0.000 \\
$P_{x} / M$ & 0.0000 & 0.1336 & 0.0000 \\
$P_{y} / M$ & -0.1336 & 0.0000 & 0.1336 \\
$P_{z} / M$ & 0.0000 & 0.0000 & 0.0000 \\
$J_{\text {rad }}^{x} / M^{2}$ & $0.236 \pm 0.007$ & $<10^{-7}$ & 0 \\
$J_{\text {rad }}^{y} / M^{2}$ & $<10^{-6}$ & $0.236 \pm 0.007$ & 0 \\
$J_{\text {rad }}^{z} / M^{2}$ & $<10^{-6}$ & $<10^{-7}$ & $0.24 \pm 0.01$ \\
\hline \hline
\end{tabular}

$30 M$. In all cases we summed over all modes with $\ell \leq 4$ 23]. The remnant hole for these configurations (SOX, S0Y, and S0Z all represent the same binary) has spin $S_{\text {remnant }} / M^{2}=0.639 \pm 0.001$ indicating that the angular momentum loss is $\delta J / M^{2}=0.237 \pm 0.001$, which agrees, to within the error estimates, with the radiated angular momenta reported in Table【.

\section{CONCLUSION}

We used the Linkage definition of the angular momentum to derive a formula for the radiated angular momentum (24), (14), (16) in terms of $\psi_{4}$. The remaining radiation formulae for the energy and linear momentum have already been given in this form in Ref. [15], Eqs. (22)-(23).

We then derived a simple formula for the radiated angular momentum based on a spin-weighted spherical harmonic decomposition of $\psi_{4}$, and have demonstrated that the formula provides the correct radiated angular momentum for binaries that radiate along all three Cartesian directions. We also compared those results with the spin of the horizon of the final black hole minus the total ADM angular momentum of the system, and find very good agreement. Note that we also used these formulae to produce this kind of check in a generic black-hole binary simulation involving unequal mass and unequal (precessing) spins, the SP6 run in Ref. [10], and obtained satisfactorily agreement. This paper thus provides practical formulae for direct application to extract radiation information from current numerical relativity simulations of compact sources.

As a final point we note that there is an ambiguity in the definition of angular momentum for generic asymptotically flat spacetimes [12, 13] that arises from the su- 
pertranslation symmetry of $\mathscr{I}^{+}$. This ambiguity can lead to inaccuracies in the computed angular momentum for generic gauges. In general one needs to confirm that the intrinsic metrics of the extraction spheres used to compute the modes of $\psi_{4}$ asymptotically approach $d s^{2}=r^{2} d \theta^{2}+r^{2} \sin ^{2} \theta d \phi^{2}$.

\section{Acknowledgments}

We thank Manuela Campanelli for providing her notes on Ref. [15] and Osvaldo Moreschi for enlightening dis- cussions. We gratefully acknowledge NSF for financial support from grant PHY-0722315. Computational resources were provided by the Funes cluster at UTB and by the Lonestar cluster at TACC.
[1] F. Pretorius, Phys. Rev. Lett. 95, 121101 (2005), grqc/0507014.

[2] M. Campanelli, C. O. Lousto, P. Marronetti, and Y. Zlochower, Phys. Rev. Lett. 96, 111101 (2006), grqc/0511048.

[3] J. G. Baker, J. Centrella, D.-I. Choi, M. Koppitz, and J. van Meter, Phys. Rev. Lett. 96, 111102 (2006), grqc/0511103.

[4] M. Campanelli, C. O. Lousto, and Y. Zlochower, Phys. Rev. D 74, 041501(R) (2006), gr-qc/0604012.

[5] J. G. Baker et al. (2006), gr-qc/0612117.

[6] A. Buonanno, G. B. Cook, and F. Pretorius (2006), grqc/0610122.

[7] H. P. Pfeiffer et al. (2007), gr-qc/0702106.

[8] M. Shibata and K. Uryu, Phys. Rev. D74, 121503 (2006), gr-qc/0612142.

[9] F. Loffler, L. Rezzolla, and M. Ansorg, Phys. Rev. D74, 104018 (2006).

[10] M. Campanelli, C. O. Lousto, Y. Zlochower, and D. Merritt (2007), gr-qc/0701164.

[11] M. Campanelli, C. O. Lousto, Y. Zlochower, and D. Merritt (2007), gr-qc/0702133.

[12] O. M. Moreschi, Class. Quant. Grav. 21, 5409 (2004), gr-qc/0209097.

[13] J. Winicour, in General Relativity and Gravitation Vol 2, edited by A. Held (Plenum, New York, 1980), pp. 71-96.
[14] M. Campanelli, B. Kelly, and C. O. Lousto, Phys. Rev. D 73, 064005 (2006), gr-qc/0510122.

[15] M. Campanelli and C. O. Lousto, Phys. Rev. D 59, 124022 (1999), gr-qc/9811019.

[16] M. Campanelli, C. O. Lousto, Y. Zlochower, B. Krishnan, and D. Merritt (2006), gr-qc/0612076.

[17] E. T. Newman and K. P. Tod, in General Relativity and Gravitation Vol 2, edited by A. Held (Plenum, New York, 1980), pp. 1-36.

[18] S. A. Teukolsky, Astrophys. J. 185, 635 (1973).

[19] M. Campanelli, C. O. Lousto, and Y. Zlochower, Phys. Rev. D 74, 084023 (2006), astro-ph/0608275.

[20] Y. Zlochower, R. Gomez, S. Husa, L. Lehner, and J. Winicour, Phys. Rev. D68, 084014 (2003), grqc/0306098.

[21] The definitions of $\partial$ and $\bar{\partial}$ in Eq. (14) differ from the conventions in 20] by a factor of -1 . We compensate for this factor in the definition of ${ }_{s} Y_{\ell m}$ in Eq. (17). Thus the ${ }_{s} Y_{\ell m}$ used here are identical to those used in [20].

[22] We have demonstrated that Eq. (30) is correct for $\left(\ell, m, \ell^{\prime}, m^{\prime}\right)$ in the range $2 \leq \ell \leq 10,-\ell \leq m \leq \ell$, $2 \leq \ell^{\prime} \leq 10$ and $-\ell^{\prime} \leq m^{\prime} \leq \ell^{\prime}$, but have not proven it in general.

[23] For these configuration the $\ell=2$ modes dominate the contribution to the radiated angular momentum. 